\title{
Jeu de miroir, cinématographie et théâtralité d'une scène stendhalienne
}

-

Murielle Rubègue

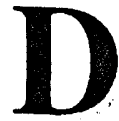

ans Le Rouge et le noir , Stendhal évoque la façon dont Julien Sorel, fils de charpentier, fait son apprentissage de la vie, et met en évidence la perpetuelle hésitation de celui-ci à choisir entre la carrière des armes et la carrière ecclésiastique. Le passage à étudier porte tout particulièrement sur le thème du regard qui peut être analysé grâce à la présence d'un jeu de miroirs. d'un aspect cinématographique et d'un aspect thêatral.

Ce passage se situe dans le chapitre 18 du Livre I, soit dans le premier quart du roman. Ce chapitre est consacré à la visite du roi à Verriere, evènement qui prend de l'ampleur dans cette petite ville de province. Après avoir defile à cheval dans la garde d'honneur afin de distraire le roi, Julien Sorel quitte l'habit militaire pour revêtir l'habit ecclésiastique et se voit chargé d'aller quérir l'évêque d'Agde à Bray-le-Haut, celui-ci y devant montrer au roi la relique de Saint Clément. L'action se passe l'après-midi du dimanche 8 septembre 1827 dans une ancienne abbaye, plus précisement dans une "immense salle gothique extrêmement. sombre et toute lambrissée de chêne noir" (133) où se trouve l'évêque d'Agde que Julien surprend en train de s'exercer à donner des bénédictions.

Dans ce passage, l'auteur met en lumière une scène de voyeurisme basée sur un jeu de miroirs. En effet, le héros entre dans une pièce sombre où il voit "un miroir mobile en acajou" (4). Le mot "miroir" est répété deux fois dans le texte (8 et 18) et deux synonymes de ce mot y apparaissent également : "la glace" (5) et "la psyche" (22). Ainsi, Julien aperçoit d'abord le miroir, puis l'évêque (de dos) qu'il ne connaît pas encore : c'est en quelque sorte une scène de première vue, mais une scène toute particulière où seul un personnage voit l'autre. En fait, Julien regarde l'évêque qui se regarde lui-même dans le miroir. On peut 
cependant remarquer que Julien est le seul personnage auquel correspond le registre du regard : "la vue" (1), "il vit" (4), "la vue fixée" et "regardant" (13), “il distinguait" (16), "Julien vit" (26). Ceci prouve donc que Julien permet au lecteur de "voir" toute cette scène. Julien voit l'évêque sans en être vu : voila tout le principe du voyeurisme qui peut aussi s'appliquer au lecteur. Le fait de voir sans être vu procure le plaisir de l'interdit, de surprendre une personne dans son intimité secrète et de violer cette intimité en toute impunité, d'où le plaisir que ressent le lecteur-voyeur grâce à Julien. En ce qui concerne l'évêque, le narrateur nous communique sa position dans l'espace -"il était arrêté à trois pas de la glace" (5) - mais à aucun moment du passage, il ne nous stipule que le prêtre regarde son reflet dans le miroir. En effet, ce personnage donne des bénédictions “du côté du miroir" (8), et lorsqu'il voit, c'est l'image de Julien et non la sienne qu'il aperçoit : "Le jeune homme le vit dans la psyche" (22). On peut conclure de cette remarque que seul ce qui est en rapport direct avec le héros importe au narrateur et donc au lecteur. Ainsi, Julien voit l'évêque qui voit sa propre image dans le miroir avant d'y voir le reflet de Julien, se retourner et voir la personne de Julien. Il s'agit donc bien d'une scène de voyeurisme, mais aussi de première vue “à retardement" dans laquelle le personnage vu aperçoit le voyeur après coup.

L'aspect cinématographique caractérisant le passage permet également de traiter la thématique du regard. En effet, dans ce passage, tout est vu par l'ocil de Julien, tel l'oeil d'une caméra. Dans la narration stendhalienne, on passe brusquement de la narration classique ou récit à la troisième personne du singulier au monologue interrieur avec discours au style direct, et vice-versa. Ainsi, dans le passage, on trouve trois monologues intérieurs ( 9 a 11, 19, 26 et 27), qui s'entrêmelent aux récit et commentaires du narrateur. On a ici une focalisation interne fixe, ou ceque Jean Pouillon appelle la "vision avec"(74) : le récit respecte la limitation d'un point de vue subjectif et observe scrupuleusement ce que Georges Blin appelle les "restrictions de champ."(105) Dans le passage, nous découvrons donc la scène avec Julien progressivement et nous n'en percevons que ce qu'il en retient. Il n'y a pas de décor planté, mais plutôt une succession de réalités perçues par Julien qui permet au lecteur de voir la scène. Comme l'a noté Jean Prévost, "presque toujours nous sommes dans l'âme du héros", "nous voyons les choses et les évènements par ses yeux."(26) Ainsi, le lecteur découvre la pièce où se trouve l'évêque en même temps que Julien : il voit d'abord les murs et leur aspect nu et mélancolique, puis "l'unique fenêtre par laquelle le jour pénètrait" (3), le miroir et enfin " un jeune homme, en robe violette et en surplis de dentelle" (4 et 5). Cette premiere étape est suivie d'un commentaire de Julien en son for intérieur (9 a 11) : celui-ci pourrait être dit à haute voix dans un film, sans que le héros n'ait à prononcer quoi que ce soit : c'est ce que l'on appelle "la voix off" dans le jargon cinématographique français. Puis nous avons une sorte de "travelling avant" (12 a 18) qui corre- 
spond à la progression de Julien dans la piece jusqu'au miroir : "il avança et parcourut assez lentement la longueur de la salle" (12), "A mesure qu'il approchait " (17), "La richesse [...] arrêta involontairement Julien à quelques pas du magnifique miroir." (17 et 18). Ensuite, nous pourrions à nouveau entendre Julien en "voix off" (19). Enfin, il y a mouvement du jeune homme, interpellation, gros plan sur lui et phénomène de reconnaissance de la part de Julien : "c'était l'évêque d'Agde" (26), précédant une dernière pensée en "voix off" (27).

Ce passage est aussi particulièrement thêatral. En effet, le propre du thêatre, c'est de confronter des acteurs sur une scène à un public qui regarde le spectacle se déroulant sous ses yeux. Dans ce passage, Julien est le témoin d'une scène étrange et vide de sens. Le décor perçu par le héros fait penser à une prison, un lieu sombre, mais d'une "magnificence mélancolique" (1) qui le "touch[e]" (2), "la beauté de la salle [...] l’ém[eut]" (19 et 20). On peut également noter limportance des couleurs : le topoi de la lumière qui pénètre par l'unique fenêtre" (3 et 13) d'où le contraste lumière-obscurité, l'omniprésence du rouge et de ses derivés (le miroir "en acajou" (4), l'acajou étant d'une couleur brun rougeâtre, la "robe violette" (4), le violet s'obtenant par le mélange du bleu et du rouge), le blanc du "surplis de dentelle" (5 et 17). Toutes ces couleurs donnent un aspect baroque au décor. De plus, le miroir revêt une importance capitale car c'est un objet "magnifique" (18), objet par excellence du narcissisme, de l'égotisme ou culte de soi, c'est pourquoi il détonne dans ce lieu religieux : "Ce meuble semblait étrange [...] et sans doute y avait été apporté de la ville." (6 et 7). C'est aussi un objet theâtral essentiel car il est au centre de la scène que répète l'évêque. En effet, l'évêque d'Agde fait une véritable mise en scène en guise de préparation avant la cérémonie : il répète son rôle avec application. D’abord, il revêt le masque de la gravité, le sérieux : "Julien trouva que le jeune homme avait l'air irrite”" (7), "il distinguait mieux son air fâche" (16), "l'air fâche”" (23). A cela s'ajoute le geste de l'évêque : "de la main droite il donnait gravement des bénédictions du côté du miroir" (8), "[il] continuait à donner des bénédictions exécutées lentement mais en nombre infini et sans se reposer un instant." (14 et 15). Ce geste thétral, saisi en gros plan, apparaît a Julien comme mystérieux : "Que peut signifier ceci ?" (9 et 10). On peut remarquer que Julien voit cela en étranger, il est le héros d'un roman de formation et est novice à cet obscur langage ecclésiastique composé de signes à déchiffrer et à apprendre : c'est un code qui signifie un au-delà du signifié apparent que seuls les initiés peuvent percevoir. Ce geste transforme l'évêque en une marionnette, un être qui l'accomplit mécaniquement et sous-entend de la part de Stendhal une critique de l'institution religieuse : un évêque joue un rôle. Les lignes 22 et 23 mettent en ćvidence le rôle que joue l'évêque : au moment où il aperçoit le reflet de Julien dans le miroir, il "quitt[e] subitement l'air fâché, lui dit du ton le plus doux." Cette attitude forme un grand contraste avec l'attitude initiale de 
l'évêque : il cesse de jouer la comédie et redevient lui-même à la grande stupéfaction du héros. (La suite du chapitre 18 révèle le pourquoi de cette scène : "Le roi de ${ }^{* * *}$ est accoutumé à un clergé vénérable et sans doute fort grave. Je ne voudrais pas, à cause de mon âge surtout, avoir l'air trop léger. [...] C'est clair, dit Julien, osant enfin comprendre, il s'exerce à donner la bénédiction." (135)).

Voyeurisme et jeu de miroirs, dimensions cinématographique et thêtrale, tels sont les différents aspects ayant suscité mon intérêt quant à la thématique du regard dont traite ce passage. La narration stendhalienne, selon moi, s'avère être d'une modernité originale, car le lecteur aime trouver dans un roman un point de vue humain et donc limité, ce qui lui permet de se transformer en personnage et de vivre une expérience imaginaire par procuration le temps dune lecture, oubliant ainsi provisoirement l'expression de Paul Valéry selon kquel les personnages ne sont que des "êtres de papier" et des "vivants sans entrailles."

University of Kansas 


\section{Ouvrages cités}

Blin, Georges. Stendhal et les problèmes du roman. Paris : Corti, 1954.

Pouillon, Jean. Temps et roman. Paris : Gallimard, 1956.

Prévost, Jean. La Création chez Stendhal. Paris : Gallimard, 1975. 
